\title{
BMJ Open Application of the iBox prognostication system as a surrogate endpoint in the TRANSFORM randomised controlled trial: proof-of-concept study
}

\author{
Olivier Aubert, ${ }^{1,2}$ Gillian Divard, ${ }^{1,2}$ Julio Pascual, ${ }^{3}$ Federico Oppenheimer, ${ }^{4}$ \\ Claudia Sommerer, ${ }^{5}$ Franco Citterio, ${ }^{6}$ Helio Tedesco, ${ }^{7}$ Steve Chadban, ${ }^{8}$ \\ Mitchell Henry, ${ }^{9}$ Flavio Vincenti, ${ }^{10}$ Titte Srinivas, ${ }^{11}$ Yoshihiko Watarai, ${ }^{12}$ \\ Christophe Legendre, ${ }^{1,2}$ Peter Bernhardt, ${ }^{13}$ Alexandre Loupy (D) 1,2
}

To cite: Aubert 0, Divard G, Pascual J, et al. Application of the iBox prognostication system as a surrogate endpoint in the TRANSFORM randomised controlled trial: proof-ofconcept study. BMJ Open 2021;11:e052138. doi:10.1136/ bmjopen-2021-052138

- Prepublication history and additional supplemental material for this paper are available online. To view these files, please visit the journal online (http://dx.doi.org/10.1136/ bmjopen-2021-052138).

$\mathrm{OA}$ and $\mathrm{GD}$ contributed equally.

Received 08 April 2021 Accepted 31 August 2021

Check for updates

(C) Author(s) (or their employer(s)) 2021. Re-use permitted under CC BY-NC. No commercial re-use. See rights and permissions. Published by BMJ.

For numbered affiliations see end of article.

Correspondence to

Dr Alexandre Loupy; alexandre.loupy@inserm.fr

\section{ABSTRACT}

Objectives Development of pharmaceutical agents in transplantation is currently limited by long waits for hard endpoints. We applied a validated integrative risk-prognostication system integrative Box (iBox) as a surrogate endpoint to the TRANSFORM Study, a large randomised controlled trial, to project individual patient long-term kidney allograft survival from 1 year to 11 years after randomisation.

Design Post-hoc analysis of a randomised open-label controlled trial.

Setting Multicentre study including 186 centres in 42 countries worldwide.

Participants 2037 de novo kidney transplant recipients. Intervention Participants were randomised (1:1) to receive everolimus with reduced-exposure calcineurin inhibitor (EVR+rCNI) or mycophenolic acid with standardexposure CNI (MPA +SCNI).

Primary outcome measure The iBox scores were computed for each participant at 1 year after randomisation using functional, immunological and histological parameters. Individual long-term deathcensored allograft survival over 4, 6 and 11 years after randomisation was projected with the iBox riskprognostication system.

Results Overall, 940 patients receiving EVR $+\mathrm{rCNI}$ and 932 receiving MPA +sCNI completed the 1-year visit. iBox scores generated at 1 year yielded graft survival prediction rates of $90.9 \%$ vs $92.1 \%, 87.9 \%$ vs $89.5 \%$, and $80.0 \%$ vs $82.4 \%$ in the EVR $+\mathrm{rCNI}$ versus MPA $+\mathrm{sCNI}$ arms at 4,6 , and 11 years post-randomisation, respectively (all differences below the 10\% non-inferiority margin defined by study protocol). Inclusion of immunological and histological Banff diagnoses parameters in iBox scores resulted in comparable and non-inferior predicted graft survival for both treatments.

Conclusions This proof-of-concept study provides the first application of a validated prognostication system as a surrogate endpoint in the field of transplantation. The iBox system, by projecting kidney allograft survival up to 11 years post-randomisation, confirms the non-inferiority of $\mathrm{EVR}+\mathrm{rCNI}$ versus MPA $+\mathrm{sCNI}$ regimen. Given the current process engaged for surrogate endpoints qualification, this

\section{STRENGTHS AND LIMITATIONS OF THIS STUDY}

$\Rightarrow$ Proof-of-concept study of the first application of a validated risk-prognostication system (integrative Box/iBox) in the large randomised controlled trial TRANSFORM in kidney transplantation.

$\Rightarrow$ The iBox prognostication system previously reported is a validated surrogate endpoint of longterm death-censored allograft failure after kidney transplantation.

$\Rightarrow$ Projection of the long-term risk of allograft failure up to 11 years post-randomisation with the 1-year post-randomisation validated data.

$\Rightarrow$ Anti-human leucocyte antigen donor-specific antibodies (HLA DSA) and allograft biopsy part of the original iBox score were not in the protocol of the TRANSFORM Study.

$\Rightarrow$ Derived iBox scores were used to take into account missing variables at 1 year (anti-HLA DSA and allograft biopsy).

study illustrates the potential to fast track development of pharmaceutical agents.

Trial registration number TRANSFORM trial:

NCT01950819.

iBox prognostication system: NCT03474003.

\section{INTRODUCTION}

Despite improvement in short-term allograft survival outcomes following renal transplantation, long-term allograft survival remains a challenge, ${ }^{12}$ and prediction of long-term outcomes suffers from lack of studies with statistical power due to the low event rates of graft failure and mortality during the first year post-transplantation. ${ }^{2-4}$ Attention has been made on defining early surrogate endpoints that would serve for therapeutic interventions, clinical trials and clinical decisionmaking. ${ }^{4-10}$ This pressing need has been raised by the transplant societies (European 
Society of Organ Transplantation, American Society for Transplantation and the American Society of Transplant Surgeons), agencies (European Medicine Agency and Food and Drug Administration), and consortia. ${ }^{11} 12$ Indeed, the lack of early surrogate endpoints is a serious threat to drug discovery and a limitation to innovation in providing improvements of kidney allograft survival.

The accuracy of graft survival prediction based on individual parameters such as estimated glomerular filtration rate (eGFR) ${ }^{10}{ }^{13} 14$ proteinuria,${ }^{15}$ histology ${ }^{16}{ }^{17}$ or human leucocyte antigen (HLA) antibody profiles ${ }^{17-19}$ is poor because individual parameters do not take into account the complete phenotype of a patient.

The integrative Box (iBox) scoring system was the first risk-prediction system in renal transplantation to be developed and validated using outcomes data from 10 centres in Europe and North America and from three randomised controlled trials (RCTs).${ }^{20}$ The iBox scoring system integrating functional (eGFR and proteinuria), histological, and immunological factors evaluated at any time point post-transplant has exhibited robust calibration and discrimination as assessed by concordance statistics in the derivation and validation cohorts from Europe and the USA. ${ }^{20}$ The iBox system was also independently validated with outcomes data from three registered phase 2 and 3 trials, and three additional studies on antibody-mediated rejection (AMR), T cell-mediated rejection (TCMR) and calcineurin inhibitor (CNI)sparing regimen studies, where iBox scores and projected allograft survival rates calculated for all patients in the trials were compared with the actual allograft failure rates and found to reveal accurate discrimination overall, thereby confirming its ability to accurately predict longterm allograft survival in RCTs early after transplantation or randomisation..$^{20}$ Additionally, the iBox system has shown sufficient evidence of its performance as a surrogate endpoint for long-term allograft survival to be accepted in the biomarker qualification programme from the Center for Drug Evaluation and Research of the US Food and Drug Administration. ${ }^{21}$

TRANSFORM was the largest, multicentre, intent-totreat trial in de novo renal transplantation to employ a binary composite endpoint assessing immunosuppressive efficacy and graft function as a surrogate for longterm graft survival. ${ }^{22}$ The study findings demonstrated that everolimus with reduced-exposure CNI $(\mathrm{EVR}+\mathrm{rCNI})$ regimen was non-inferior (using a $10 \%$ non-inferiority margin) to mycophenolic acid with standard-exposure CNI (MPA + sCNI) regimen for the binary endpoint, with stable renal function and no new safety signals up to 2 years post-transplantation. ${ }^{23}{ }^{24}$ Even though this study showed no differences in anti-rejection efficacy and renal function outcomes between the two treatments, it was limited by the relatively short follow-up after randomisation and the low event rates of graft loss and mortality. ${ }^{24}$

Here, we show the first application of a validated risk-prognostication system to a single study with $>2000$ randomised patients via individual risk evaluation at
1 year and predict long-term death-censored allograft survival probability up to 11 years post-randomisation.

\section{METHODS}

Participants

In the TRANSFORM Study, 2037 de novo renal transplant recipients were randomised (1:1) within 24 hours of transplantation to receive either EVR $+\mathrm{rCNI}$ or MPA $+\mathrm{sCNI}$, in combination with induction therapy and corticosteroids. The primary endpoint comprised a binary composite of incidence of treated biopsy-proven acute rejection or eGFR $<50 \mathrm{~mL} / \mathrm{min} / 1.73 \mathrm{~m}^{2}$ at 1 year post-transplantation using a $10 \%$ non-inferiority margin. ${ }^{23}$ Eligibility criteria of patients, induction therapy and immunosuppression regimens used, including protocol-defined target trough levels of EVR and CNIs (tacrolimus and cyclosporine) have been previously described. ${ }^{23}$

\section{Allograft loss risk prediction using iBox scores}

Individual allograft survival probabilities were determined using the iBox scoring system. ${ }^{20}$ Data from patients completing the 1-year visit were included for generation of iBox risk-prediction scores after confirming the availability of functional, immunological, and/or histological follow-up data. The iBox scores were generated using eight parameters, including baseline characteristics (time from transplant to evaluation), functional parameters (eGFR and urine protein/creatinine ratio), immunological parameters (presence of circulating anti-HLA donorspecific antibodies (DSA)), and histological parameters (biopsy findings including microcirculation inflammation (glomerulitis Banff score and peritubular capillaritis Banff score, interstitial inflammation and tubulitis Banff scores, transplant glomerulopathy Banff score, and interstitial fibrosis/tubular atrophy Banff score)). To account for missing variables and allow adaptation at individual centres, a derived score using the diagnoses of AMR, TCMR, BK virus-associated nephropathy, or recurrence of end-stage renal disease (ESRD) instead of Banff scores was generated and previously reported. ${ }^{20}$ Thus, three types of risk scores were generated based on available parameters and to allow flexibility across centres:

- iBox ${ }^{\# 1}$ : functional (time from transplant to evaluation +eGFR+proteinuria) score.

- iBox $^{\# 2}$ : functional +immunological (DSA) score.

- iBox ${ }^{\# 3}$ : functional +immunological+histological (biopsy diagnoses) score.

\section{Measurements performed at 1 year}

Kidney allograft function was assessed by eGFR as estimated by the four-variable modification of diet in renal disease equation and by proteinuria $(\mathrm{g} / \mathrm{g})$ as estimated by the urinary protein/creatinine ratio (UPCR). Circulating DSA against HLA-A, HLA-B, and HLA-DR, and histological diagnoses data were assessed according to local centre practice. 


\section{Outcomes}

The outcome of interest was the projection of long-term death-censored allograft survival of individual patients at 3, 5,7 , and 10 years post-evaluation, which were equivalent to $4,6,8$, and 11 years post-randomisation/transplantation, respectively, using the iBox scoring system and the comparison of the mean projected allograft survival in the two arms using a $10 \%$ non-inferiority margin. The $10 \%$ margin was chosen for consistency with the primary study. ${ }^{23}$

\section{Sensitivity analyses}

To test the robustness of the iBox risk score performed at 1 year post-randomisation, the iBox risk score was applied on the measurements performed at 2years post-randomisation. ${ }^{24}$

\section{Statistical analysis}

Continuous variables were reported as mean (SD). Mean values and proportions between groups were compared using Student's t-test or the $\mathrm{X}^{2}$ test (or Fisher's exact test if appropriate). All tests were two-tailed and a $\mathrm{p}$ value of $<0.05$ was considered to be statistically significant. The iBox risk-prediction scores were calculated for each patient according to the $\beta$-regression coefficients of the iBox model as previously published. ${ }^{20}$ Death-censored allograft survival probabilities at 3,5, 7 and 10 years postevaluation were determined after adjustment for actual allograft survival rates in both the arms at 1 year. All analyses were performed using $\mathrm{R}$ (V.3.2.1, R Foundation for Statistical Computing), all the packages used are detailed in the online supplemental methods.

\section{Patient and public involvement}

The iBox prognostication system potential for patient care and clinical trials was presented and discussed among the two main French patients' associations, involving patients, nurses and healthcare professionals.

\section{RESULTS}

\section{Patient disposition and baseline characteristics}

In the TRANSFORM Study, 2037 de novo renal transplant recipients were randomised between December 2013 and January 2016 and comprised the intent-to-treat population (EVR +rCNIarm, N=1022 and MPA +sCNIarm, $\mathrm{N}=1015) .{ }^{23}$ Of these, $940(92.0 \%)$ patients in the $\mathrm{EVR}+\mathrm{rCNI}$ arm and $932(91.8 \%)$ in the MPA + sCNI arm who had completed the 1-year visit underwent iBox risk evaluation. Demographics and baseline characteristics of patients who underwent risk evaluation were well balanced in both treatment arms (table 1).

\section{Functional and immunological parameters at 1 year post-}

\section{randomisation}

Mean eGFR at 1 year was comparable in the $\mathrm{EVR}+\mathrm{rCNI}$ and MPA +sCNI arms (55.6 (19.95) vs 56.2 (18.97) $\mathrm{mL} / \mathrm{min} / 1.73 \mathrm{~m}^{2} \quad(\mathrm{p}=0.4864$; online supplemental figure 1). The mean UPCR was $0.32(0.67) \mathrm{g} / \mathrm{g}$ for the EVR +rCNIarm and 0.25 (0.58) $\mathrm{g} / \mathrm{g}$ for the
MPA +sCNIarm ( $\mathrm{p}=0.0134$; online supplemental figure 2).

Among patients with DSA assessment at 1 year, 56 of $408(13.7 \%)$ were positive for DSA in the EVR + rCNI arm compared with 62 of $392(15.8 \%)$ in the MPA + sCNI arm $(\mathrm{p}=0.404)$.

\section{Histological diagnoses at 1 year post-randomisation}

Histological parameters were assessed in all patients who had biopsy evaluation during the first year postrandomisation. Incidence of active and chronic AMR, TCMR, borderline rejections, BK virus-associated nephropathy and recurrence of ESRD were not significantly different between the two treatment arms (online supplemental table 1).

\section{iBox scores at 1 year post-randomisation}

A total of $940(92.0 \%)$ patients in the EVR +rCNIarm and $932(91.8 \%)$ in the MPA +sCNI arm underwent risk evaluation at 1 year. After excluding 14 patients in each arm without proteinuria data, iBox ${ }^{\# 1}$ scores were generated for $926(98.5 \%)$ and $918(98.5 \%)$ patients in the $\mathrm{EVR}+\mathrm{rCNI}$ and MPA $+\mathrm{sCNI}$ arms, respectively. iBox $^{\# 2}$ scores were generated for 405 patients $(43.1 \%)$ in the EVR +rCNIarm and 388 patients (41.6\%) in the MPA +sCNIarm for whom DSA parameters were also available, and BBox $^{\# 3}$ scores were generated for 177 $(18.8 \%)$ and $178(19.1 \%)$ patients in the EVR +rCNI and MPA +sCNI arms, respectively, who had all functional, immunological, and histological parameters available (figure 1).

\section{iBox application and projected long-term death-censored allograft survival}

The mean iBox $^{\# 1}$ scores at 1 year were -3.28 (1.09) for the $\mathrm{EVR}+\mathrm{rCNI}$ arm and -3.42 (1.06) for the MPA +sCNI arm (figure 2A). These risk scores translated into mean projected allograft survival rates of $90.9 \%, 87.9 \%, 84.3 \%$, and $80.0 \%$ for the EVR +rCNI arm and $92.1 \%, 89.5 \%$, $86.2 \%$, and $82.4 \%$ for the MPA + sCNI arm at 3, 5, 7 and 10 years post-evaluation (or 4, 6, 8 and 11 years postrandomisation), respectively. Thus, a difference of $2.4 \%$ (lower than the $10 \%$ non-inferiority margin) in favour of the MPA + sCNI regimen was projected at 10 years postevaluation (figure 2B). This result remains consistent when we stratified by donor type (online supplemental figures 3 and 4 ).

The mean iBox ${ }^{\# 2}$ scores at 1 year were -3.13 (1.06) and -3.23 (1.06) for the EVR +rCNI and MPA +sCNI arms, respectively (figure $3 \mathrm{~A}$ ). The mean projected allograft survival rates using iBox $^{\# 2}$ scores at 3, 5, 7 and 10 years post-evaluation were $90.6 \%, 87.5 \%, 83.6 \%$ and $78.9 \%$ for the $\mathrm{EVR}+\mathrm{rCNI}$ arm and $91.6 \%, 88.7 \%, 85.2 \%$, and $80.9 \%$ for the MPA $+\mathrm{sCNI}$ arm, corresponding to a non-inferior difference of $2.0 \%$ at 10 years (under the $10 \%$ margin) in favour of the MPA + sCNI regimen (figure $3 \mathrm{~B}$ ).

The mean iBox $^{\# 3}$ scores at 1 year were -2.57 (1.18) for the EVR +rCNIarm and -2.84 (1.08) for the 
Table 1 Demographics and baseline characteristics of patients at iBox risk evaluation

\begin{tabular}{|c|c|c|c|c|}
\hline & $\mathbf{N}$ & $\begin{array}{l}\text { EVR +rCNI } \\
\mathrm{N}=940\end{array}$ & $\begin{array}{l}\text { MPA+sCNI } \\
\mathrm{N}=932\end{array}$ & $P$ value \\
\hline \multicolumn{5}{|l|}{ Recipients' characteristics } \\
\hline Age, $n(\%)$ & 1871 & & & 0.571 \\
\hline $18-30$ years & 633 & 328 (34.9) & $305(32.7)$ & \\
\hline $31-55$ years & 1065 & $529(56.3)$ & $536(57.6)$ & \\
\hline $56-80$ years & 173 & $83(8.8)$ & $90(9.7)$ & \\
\hline Male sex & & $652(69.4)$ & $646(69.3)$ & 0.982 \\
\hline Cold ischaemia time, hours, mean (SD) & 1859 & $8.1(7.7)$ & $8.2(7.7)$ & 0.820 \\
\hline Delayed graft function, $\mathrm{n}(\%)$ & 174 & $93(9.9)$ & $81(8.7)$ & 0.370 \\
\hline HLA-A/B/DR mismatch, mean (SD) & 1862 & $3.4(1.5)$ & $3.4(1.5)$ & 0.620 \\
\hline Type of CNI, n (\%) & 1868 & & & 0.836 \\
\hline Tacrolimus & & $846(90.4)$ & $845(90.7)$ & \\
\hline Cyclosporine & & $90(9.6)$ & $87(9.3)$ & \\
\hline Induction, $\mathrm{n}(\%)$ & 1871 & & & 0.795 \\
\hline Basiliximab & & $789(84.0)$ & $779(83.6)$ & \\
\hline rATG & & $150(16.0)$ & $153(16.4)$ & \\
\hline ESRD leading to transplant & 1872 & & & 0.885 \\
\hline Glomerulonephritis & & $144(15.3)$ & $154(16.5)$ & \\
\hline Diabetes & & $118(12.6)$ & 120 (12.9) & \\
\hline Hypertension/nephrosclerosis & & $115(12.2)$ & $114(12.2)$ & \\
\hline Other & & $427(45.4)$ & $423(45.4)$ & \\
\hline Unknown & & $136(14.5)$ & $121(13.0)$ & \\
\hline \multicolumn{5}{|l|}{ Donor characteristics, n (\%) } \\
\hline Male sex & 869 & 423 (48.9) & $446(51.4)$ & 0.291 \\
\hline Donor category & 1717 & & & 0.329 \\
\hline Living related & & $270(31.6)$ & 249 (28.9) & \\
\hline Living unrelated & & $173(20.2)$ & $161(18.7)$ & \\
\hline Deceased non-heart beating & & $3(0.4)$ & $5(0.6)$ & \\
\hline Deceased heart beating & & $409(47.8)$ & 447 (51.8) & 0.084 \\
\hline Standard criteria donor & & $295(72.1)$ & $298(66.7)$ & \\
\hline Expanded criteria donor & & $114(27.9)$ & $149(33.3)$ & \\
\hline
\end{tabular}

CNI, calcineurin inhibitor; ESRD, end-stage renal disease; EVR, everolimus; HLA, human leucocyte antigen; iBox, integrative Box; MPA, mycophenolic acid; rATG, rabbit antithymocyte globulin; rCNI, reduced-exposure CNI; sCNI, standard-exposure CNI.

MPA + sCNI arm (figure 4A). These scores yielded mean projected allograft survival rates of $87.5 \%, 83.3 \%, 78.3 \%$, and $72.4 \%$ for the EVR +rCNI arm and $90.4 \%, 87.1 \%$, $83.0 \%$, and $78.1 \%$ for the MPA +sCNI arm, respectively, corresponding to a difference of $5.7 \%$ at 10 years (under the $10 \%$ margin) in favour of the MPA + sCNI regimen (figure 4B).

\section{Sensitivity analyses}

A total of $893(95.0 \%)$ patients in the EVR + rCNIarm and $881(94.5 \%)$ in the MPA +sCNI arm underwent risk evaluation at 1 and 2 years post-randomisation. When we applied the $\operatorname{iBox}^{\# 1}$ (functional) score on the 2-year measurements (time from transplant to evaluation,
eGFR and proteinuria), the results remained consistent: the mean BBox $^{\# 1}$ scores at 2 years were -3.11 (0.04) for the EVR +rCNI arm and -3.29 (0.04) for the MPA + sCNI arm (online supplemental figure 5A). These risk scores translated into mean projected allograft survival rates of $89.3 \%, 85.6 \%, 81.5 \%$, and $76.7 \%$ for the EVR + rCNI arm and $90.7 \%, 87.7 \%, 84.1 \%$, and $78.9 \%$ for the MPA +sCNI arm at 3, 5, 7 and 10 years post-evaluation (or 5, 7, 9 and 12 years post-randomisation), respectively. Thus, a difference of $3.2 \%$ (lower than the $10 \%$ noninferiority margin) in favour of the MPA $+\mathrm{sCNI}$ regimen was projected at 10 years post-evaluation (online supplemental figure $5 \mathrm{~B}$ ). 


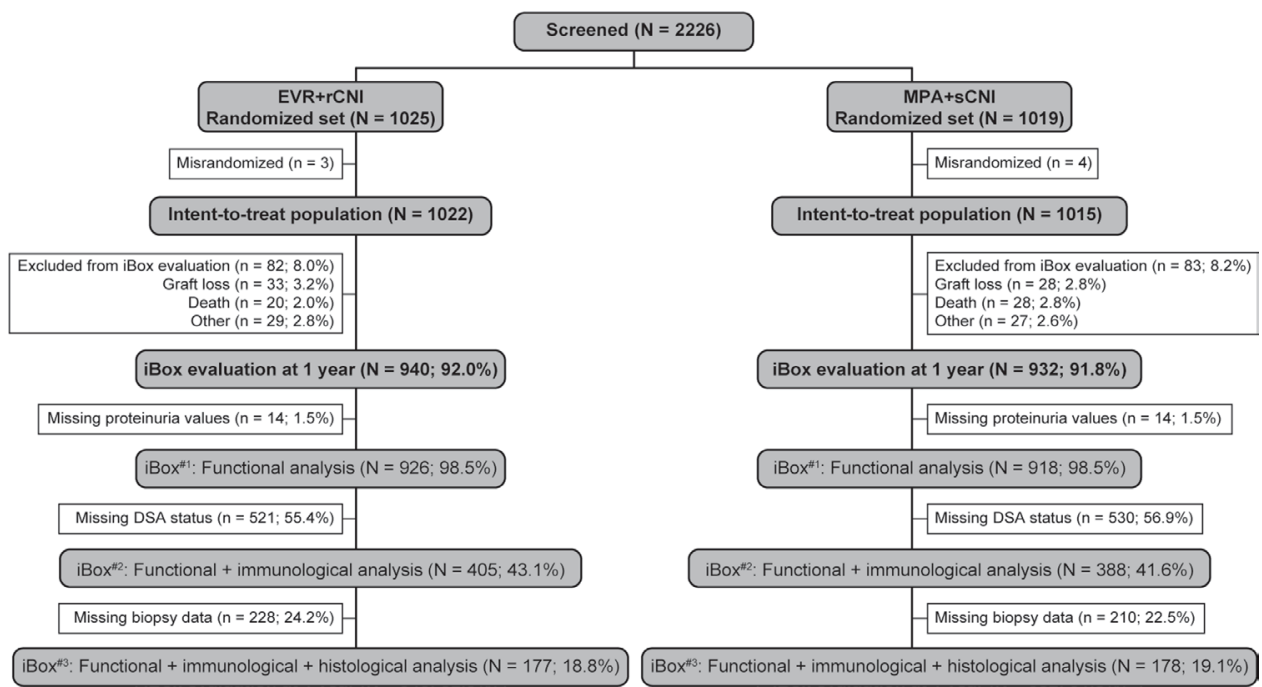

Figure 1 Patient disposition. DSA, donor-specific antibodies; EVR, everolimus; iBox, integrative Box; MPA, mycophenolic acid; rCNI, reduced-exposure calcineurin inhibitor; sCNI, standard-exposure calcineurin inhibitor.

\section{DISCUSSION}

This study is the first application of a validated multidimensional risk scoring system iBox to predict long-term allograft survival in an RCT.

In TRANSFORM, an EVR +rCNI regimen was found to be non-inferior in terms of efficacy to a standardof-care regimen comprising MPA + sCNI up to 2 years post-transplantation. ${ }^{22} 23$ Using individual risk scores of patients from the study at 1 year, we found that mean projected allograft survival rates with both the regimens were comparable at $3,5,7$, and 10 years post-evaluation or $4,6,8$, and 11 years post-randomisation, thereby reinforcing the 1-year and 2-year actual findings for graft survival. $^{23}$ The non-inferiority margin was met for survival projections with three types of iBox scores with varying availability of functional (eGFR and proteinuria), immunological (presence of anti-HLA DSA), and histological (biopsy) parameters at evaluation, despite between-arm differences in rates. Additionally, these results remained consistent when we applied the iBox on the 2-year measurements.

This study is also the first application of iBox as a surrogate endpoint for an RCT that was originally designed to have a surrogate endpoint for long-term allograft survival, thereby increasing its applicability to other RCTs.

The iBox scores were generated using simple parameters that are broadly used by the transplant community as routine clinical practice, thereby increasing their

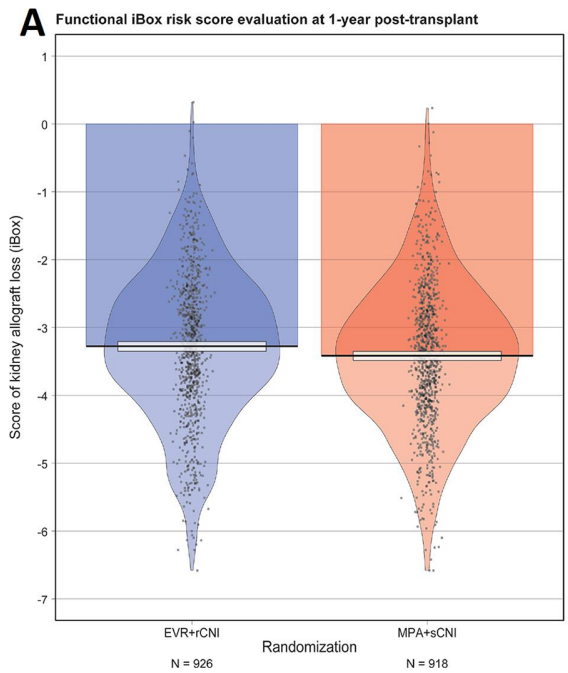

B

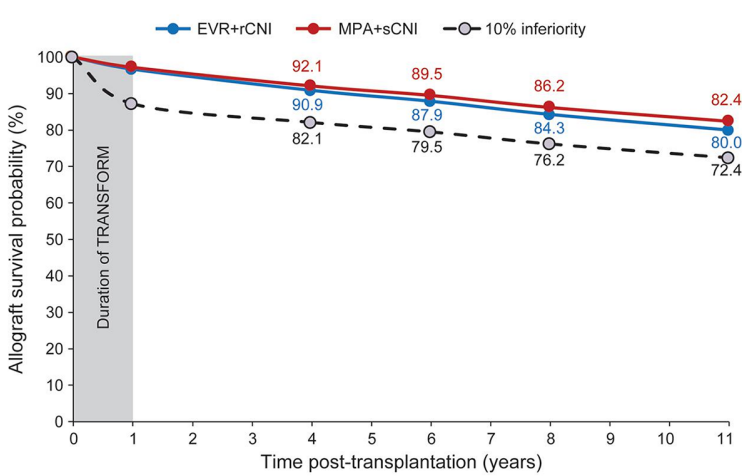

Figure 2 iBox ${ }^{\# 1}$ scores at 1 year after randomisation and projected allograft survival. (A) Mean iBox ${ }^{\# 1}$ scores in the $\mathrm{EVR}+\mathrm{rCNI}$ and MPA $+\mathrm{sCNI}$ arms at 1 year. (B) Projected allograft survival in EVR +rCNI arm (blue line) and MPA $+\mathrm{sCNI}$ arm (red line). The black dotted line corresponds to the $10 \%$ inferiority margin. The duration of TRANSFORM is the time between randomisation and the endpoint of the TRANSFORM Study (1 year post-randomisation). The projected kidney allograft survival takes into account the event (death-censored graft loss) that occurred during the duration of TRANSFORM Study. EVR, everolimus, iBox, integrative Box; MPA, mycophenolic acid; rCNI, reduced-exposure calcineurin inhibitor; sCNI, standardexposure calcineurin inhibitor. 
A

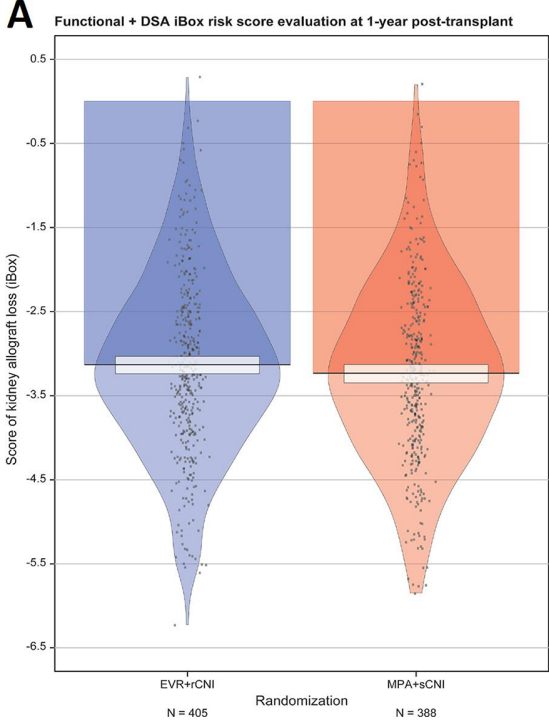

B

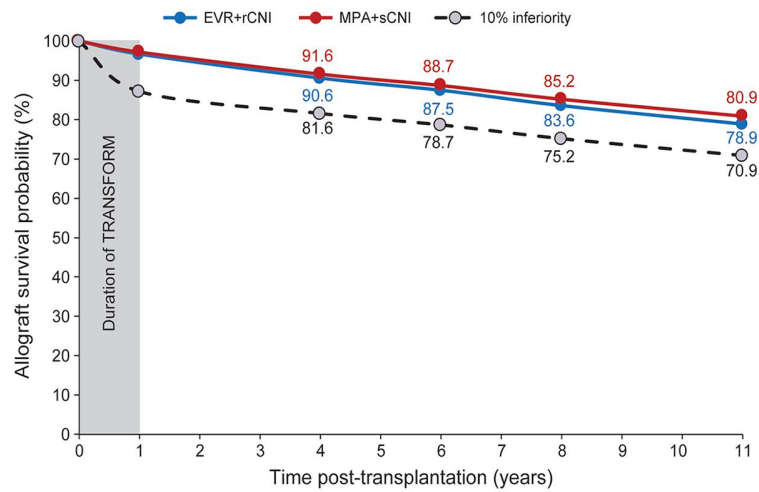

Figure $3 \mathrm{iBox}^{\# 2}$ scores at 1 year after randomisation and projected allograft survival. (A) Mean iBox ${ }^{\# 2}$ scores in the EVR $+\mathrm{rCNI}$ and MPA $+\mathrm{sCNI}$ arms at 1 year. (B) Projected allograft survival in EVR $+\mathrm{rCNI}$ arm (blue line) and MPA $+\mathrm{sCNI}$ arm (red line). The black dotted line corresponds to the $10 \%$ inferiority margin. The duration of TRANSFORM is the time between randomisation and the endpoint of the TRANSFORM Study (1 year post-randomisation). The projected kidney allograft survival takes into account the event (death-censored graft loss) that occurred during the duration of TRANSFORM Study. DSA, donorspecific antibodies; EVR, everolimus; iBox, integrative Box; MPA, mycophenolic acid; rCNI, reduced-exposure calcineurin inhibitor; sCNI, standard-exposure calcineurin inhibitor.

generalisability across centres. The association of functional, immunological and histological parameters allows for robust predictions taking into consideration the complete phenotype of a patient instead of only the eGFR, which alone is a poor predictor allograft survival. ${ }^{10} 1314 \mathrm{In}$ previous validation of iBox with cohorts of $>7000$ patients, a graft failure rate of $14.1 \%$ was observed after a median post-transplant follow-up of 7.12 years, which corresponds to a graft survival rate of $85.9 \% .^{20}$ This rate is consistent with the projected graft survival rate for TRANSFORM at 7 years when functional iBox scores were used (EVR +rCNIvs MPA +sCNI: $84.3 \%$ vs $86.2 \%$ ). Thus, the findings indicate applicability of iBox to other RCTs.

The strength of the study is that it does not compare the mean of one or several variables, which can be misleading due to non-linear association with graft survival outcome.
A Functional + DSA + histological diagnoses iBox risk score

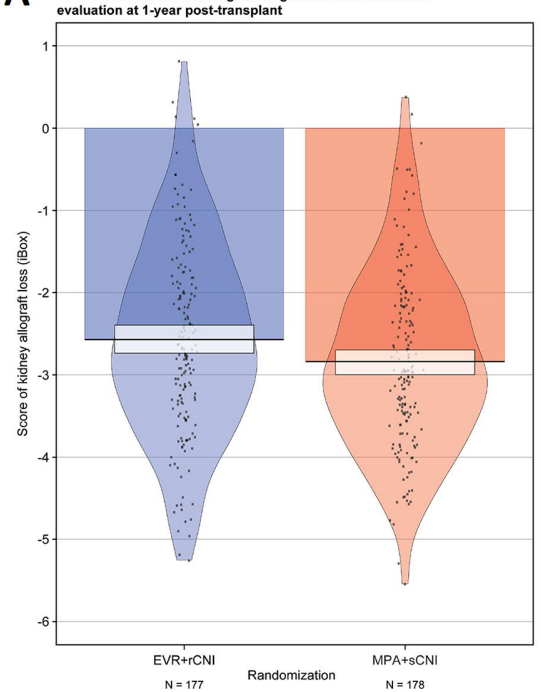

B

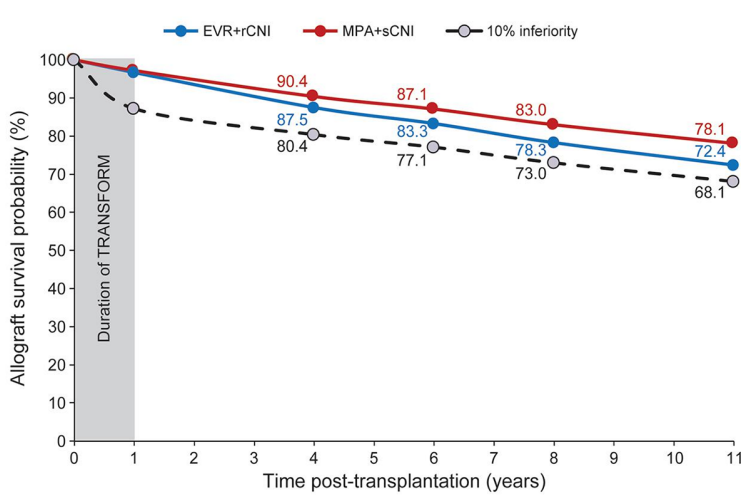

Figure 4 iBox ${ }^{\# 3}$ scores at 1 year after randomisation and projected allograft survival. (A) Mean iBox ${ }^{\# 3}$ scores in the $\mathrm{EVR}+\mathrm{rCNI}$ and MPA $+\mathrm{sCNI}$ arms at 1 year. (B) Projected allograft survival in EVR $+\mathrm{rCNI}$ arm (blue line) and MPA $+\mathrm{sCNI}$ arm (red line). The black dotted line corresponds to the $10 \%$ inferiority margin. The duration of TRANSFORM is the time between randomisation and the endpoint of the TRANSFORM Study (1 year post-randomisation). The projected kidney allograft survival takes into account the event (death-censored graft loss) that occurred during the duration of TRANSFORM Study. DSA, donorspecific antibodies; EVR, everolimus, iBox, integrative Box; MPA, mycophenolic acid; rCNI, reduced-exposure calcineurin inhibitor; sCNI, standard-exposure calcineurin inhibitor. 
For TRANSFORM, the iBox score was generated for each patient available for evaluation at 1 year and an individual projected allograft survival rate was calculated, taking into account the distribution of scores in each arm and not just absolute values.

We acknowledge that the main limitation of this study was the varying survival prediction rates obtained using iBox $^{\# 1}$, iBox $^{\# 2}$ and iBox ${ }^{\# 3}$ scores because of absence of all iBox components at 1 year. In this regard, it is noteworthy that DSA measurements were performed at 1 and 2 years for only a subset of consenting patients $(<50 \%) .{ }^{24}$ One-year biopsies were available for $<20 \%$ of patients because they were not protocol mandated, ${ }^{22}$ without central pathology reading, and performed for-cause, that is, in cases of impaired renal function and presence of proteinuria and/or de novo DSA, which could have led to selection bias. Moreover, the Banff histological diagnoses are less granular than the Banff scores even if the iBox has shown good performance using the Banff histological diagnoses instead of the Banff scores. Thus, the difference in the subset of patients with for-cause biopsies was anticipated. Furthermore, a centre effect cannot be excluded in the way biopsies were conducted. This is a limitation inherent to the TRANSFORM Study, which was not primarily designed to use iBox as a surrogate endpoint. Despite this limitation, the results were consistent with the scores generated using functional parameters and functional and immunological parameters meaning that the bias did not introduce any asymmetry between the cohorts in terms of number, characteristics and risk assessment. Regardless of the type of iBox score, the overall absolute between-arm difference in terms of long-term allograft survival projected by the three types of scores remained in the same range. While we note that projections of survival need to be validated by real-life experience, we would submit that our findings serve an important unmet need, that is, a window into long-term survival based on stringent patient-level observations obtained in the context of an RCT that used an endpoint that integrated both freedom from rejection and eGFR in a non-inferiority design in one of the largest clinical trials in kidney transplantation. Further, these observations are accrued in the context of a discrete follow-up period, thereby addressing the practical constraints of prolonged long-term follow-up that is needed to measure actual accrued outcomes. Last, the iBox score was generated in a cohort with most of the patients receiving CNI, either as reduction regimen with EVR or standard regimen with MPA. Thus, the prognostic weighting of proteinuria under EVR-based regimen and its impact on iBox score and subsequent allograft survival prediction will need further investigation. However, the iBox scoring system has been previously validated as a surrogate endpoint in the CERTITEM (NCT01079143) Study with an EVR-based regimen. ${ }^{25}$ This study was a randomised, open-label, multicentre trial that compared a CNI-based regimen with an EVR-based regimen in kidney recipients. The iBox scores were computed with the 1-year measurement (initial primary endpoint of this study) in the intention-to-treat population and compared with the actual allograft failures. The predictive performances of the iBox system in this study were high in terms of discrimination and calibration, thereby validating that the iBox can be used as a surrogate endpoint in an RCT with an EVR-based regimen. ${ }^{20}$

In conclusion, the iBox system confirms good allograft survival and the non-inferiority of EVR with reduced CNI exposure versus MPA with standard CNI exposure in terms of allograft survival up to 11 years after patient randomisation. Given the unmet need for surrogate endpoints in clinical trials, the study findings of this proof-of-concept study indicate that iBox could potentially serve as a clinical trial simulation tool to fast track the development and approval of pharmaceutical agents.

\section{Author affiliations}

${ }^{1}$ University of Paris, INSERM, PARCC, Paris Translational Research Centre for Organ Transplantation, Paris, France

${ }^{2}$ Kidney Transplant Department, Necker Hospital, APHP, Paris, France

${ }^{3}$ Department of Nephrology, Hospital del Mar, Barcelona, Spain

${ }^{4}$ Department of Nephrology and Renal Transplantation, Renal Transplant Unit, Hospital Clinic de Barcelona, Barcelona, Spain

${ }^{5}$ Department of Nephrology, University Hospital Heidelberg, Heidelberg, Germany ${ }^{6}$ Agostino Gemelli University Polyclinic Foundation, Catholic University of the Sacred Heart, Milan, Italy

${ }^{7}$ Nephrology Division, Hospital do Rim, UNIFESP, Sao Paulo, Brazil

${ }^{8}$ Department of Renal Medicine and Transplantation, Royal Prince Alfred Hospital, Camperdown, New South Wales, Australia

${ }^{9}$ Department of Surgery, The Comprehensive Transplant Center, The Ohio State University Wexner Medical Center, Columbus, Ohio, USA

${ }^{10}$ Department of Surgery, Kidney Transplant Service, University of California San Francisco, San Francisco, California, USA

${ }^{11}$ Division of Nephrology and Hypertension, University Hospitals of Cleveland, Cleveland, Ohio, USA

${ }^{12}$ Department of Transplant Surgery, Nagoya Daini Red Cross Hospital, Nagoya, Japan

${ }^{13}$ Department of Research and Development, Novartis, Basel, Switzerland

Acknowledgements The authors thank Prachiti Narvekar and Lakshmi Venkatraman for medical writing and editorial assistance.

Contributors $\mathrm{OA}, \mathrm{GD}$ and $\mathrm{AL}$ designed the study, analysed and interpreted the data, and wrote and edited the manuscript. JP, FO, CS, FC, HT, SC, MH, FV, TS, YW, $\mathrm{CL}$ and $\mathrm{PB}$ interpreted the data and edited the manuscript. All authors approved the final version of the manuscript.

Funding This work was supported in part by French national research agency (INSERM) ATIP Avenir and Fondation Bettencourt Schueller and in part by Novartis as a grant.

Competing interests JP, FO, CS, FC, HT, SC, MH, FV, TS, YW and CL comprised the scientific steering committee of TRANSFORM Study. FO has received speaker fees and travel support from Novartis. HT has received educational and travel grants from Novartis. PB is an employee of Novartis. AL holds shares in Cibiltech, a company that builds software.

Patient consent for publication Not required.

Ethics approval The study protocol CRAD001A2433 of the TRANSFORM Study was approved by the Institutional Review Board at participating centres (available in the supplemental material), Institutional Review Board (FWA\#00000068; IRB Registration 00000229), Institutional Review Board (reference number 129881; IRB Registration 14-15458), and was conducted according to the Declaration of Helsinki and the International Conference on Harmonization Guidelines for Good Clinical Practice.

Provenance and peer review Not commissioned; externally peer reviewed.

Data availability statement Data are available upon reasonable request. Technical appendix is available from the corresponding author at alexandre.loupy@aphp.fr. 
Data will be shared with investigators whose proposed use of the data has been approved by an independent review committee identified for this purpose.

Supplemental material This content has been supplied by the author(s). It has not been vetted by BMJ Publishing Group Limited (BMJ) and may not have been peer-reviewed. Any opinions or recommendations discussed are solely those of the author(s) and are not endorsed by BMJ. BMJ disclaims all liability and responsibility arising from any reliance placed on the content. Where the content includes any translated material, BMJ does not warrant the accuracy and reliability of the translations (including but not limited to local regulations, clinical guidelines, terminology, drug names and drug dosages), and is not responsible for any error and/or omissions arising from translation and adaptation or otherwise.

Open access This is an open access article distributed in accordance with the Creative Commons Attribution Non Commercial (CC BY-NC 4.0) license, which permits others to distribute, remix, adapt, build upon this work non-commercially, and license their derivative works on different terms, provided the original work is properly cited, appropriate credit is given, any changes made indicated, and the use is non-commercial. See: http://creativecommons.org/licenses/by-nc/4.0/.

ORCID iD

Alexandre Loupy http://orcid.org/0000-0003-3388-7747

\section{REFERENCES}

1 Coemans M, Süsal C, Döhler B, et al. Analyses of the short- and long-term graft survival after kidney transplantation in Europe between 1986 and 2015. Kidney Int 2018;94:964-73.

2 Hart A, Smith JM, Skeans MA, et al. OPTN/SRTR 2017 annual data report: kidney. Am J Transplant 2019;19 Suppl 2:19-123.

3 Fergusson NA, Ramsay T, Chassé M, et al. Impact of using alternative graft function endpoints: a secondary analysis of a kidney transplant trial. Transplant Direct 2019;5:e439.

4 Maggiore U, Leventhal J, Cravedi P. Rethinking clinical endpoints in kidney transplant trials. Curr Opin Organ Transplant 2020;25:1-7.

5 Shabir S, Halimi J-M, Cherukuri A, et al. Predicting 5-year risk of kidney transplant failure: a prediction instrument using data available at 1 year posttransplantation. Am J Kidney Dis 2014;63:643-51.

6 Stamenic D, Rousseau A, Essig M, et al. A prognostic tool for individualized prediction of graft failure risk within ten years after kidney transplantation. J Transplant 2019;2019:1-10.

7 Galichon P, Xu-Dubois Y-C, Finianos S, et al. Clinical and histological predictors of long-term kidney graft survival. Nephrol Dial Transplant 2013;28:1362-70.

8 Sautenet B, Tong A, Chapman JR, et al. Range and consistency of outcomes reported in randomized trials conducted in kidney transplant recipients: a systematic review. Transplantation 2018;102:2065-71.

9 Hariharan S, Kasiske B, Matas A, et al. Surrogate markers for longterm renal allograft survival. Am J Transplant 2004;4:1179-83.

10 Kasiske BL, Israni AK, Snyder JJ, et al. The relationship between kidney function and long-term graft survival after kidney transplant. Am J Kidney Dis 2011;57:466-75.
11 Stegall MD, Troy Somerville K, Everly MJ, et al. The importance of drug safety and tolerability in the development of new immunosuppressive therapy for transplant recipients: the transplant therapeutics Consortium's position statement. Am J Transplant 2019;19:625-32.

12 US Food \& Drug Administration. Surrogate endpoint resources for drug and biologic development. Available: https://www.fda.gov/ drugs/development-resources/surrogate-endpoint-resources-drugand-biologic-development [Accessed 25 Sep 2020].

13 Kaplan B, Schold J, Meier-Kriesche H-U. Poor predictive value of serum creatinine for renal allograft loss. Am J Transplant 2003;3:1560-5

$14 \mathrm{He}$ X, Moore J, Shabir S, et al. Comparison of the predictive performance of eGFR formulae for mortality and graft failure in renal transplant recipients. Transplantation 2009;87:384-92.

15 Naesens M, Lerut E, Emonds M-P, et al. Proteinuria as a noninvasive marker for renal allograft histology and failure: an observational cohort study. J Am Soc Nephrol 2016;27:281-92.

16 Yilmaz S, Tomlanovich S, Mathew T, et al. Protocol core needle biopsy and histologic chronic allograft damage index (CADI) as surrogate end point for long-term graft survival in multicenter studies. J Am Soc Nephrol 2003;14:773-9.

17 Gonzales MM, Bentall A, Kremers WK, et al. Predicting individual renal allograft outcomes using risk models with 1-year surveillance biopsy and alloantibody data. J Am Soc Nephrol 2016;27:3165-74.

18 Lefaucheur C, Loupy A, Hill GS, et al. Preexisting donor-specific HLA antibodies predict outcome in kidney transplantation. J Am Soc Nephrol 2010;21:1398-406.

19 Prémaud A, Filloux M, Gatault P, et al. An adjustable predictive score of graft survival in kidney transplant patients and the levels of risk linked to de novo donor-specific anti-HLA antibodies. PLoS One 2017;12:e0180236.

20 Loupy A, Aubert O, Orandi BJ, et al. Prediction system for risk of allograft loss in patients receiving kidney transplants: international derivation and validation study. BMJ 2019;366:14923.

21 US Food and Drug Administration. Center for drug evaluation and research (CDER) biomarker qualification program (BQP). letter of intention. Available: https://www.fda.gov/media/139300/download [Accessed 25 Sep 2020].

22 Pascual J, Srinivas TR, Chadban S, et al. Balancing efficacy and renal function preservation after kidney transplantation with everolimus and reduced calcineurin inhibitors for better graft outcomes: design of the transform study. Nephrol Dial Transpl 2014;29:iii535.

23 Pascual J, Berger SP, Witzke O, et al. Everolimus with reduced calcineurin inhibitor exposure in renal transplantation. J Am Soc Nephrol 2018;29:1979-91.

24 Berger SP, Sommerer C, Witzke O, et al. Two-Year outcomes in de novo renal transplant recipients receiving everolimus-facilitated calcineurin inhibitor reduction regimen from the transform study. Am J Transplant 2019;19:3018-34.

25 Rostaing L, Hertig A, Albano L, et al. Fibrosis progression according to epithelial-mesenchymal transition profile: a randomized trial of everolimus versus CSA. Am J Transplant 2015;15:1303-12. 